\title{
ON HOSSZÚ'S FUNCTIONAL EQUATION IN DISTRIBUTIONS
}

\author{
E. L. $\mathrm{KOH}$
}

(Communicated by Hal L. Smith)

ABSTRACT. In this paper, the Hosszú functional equation

$$
f(x+y-x y)+g(x y)=h(x)+k(y)
$$

is reformulated in the domain of distributions. Its general solution is found which includes Fenyö's solution as a special case.

\section{INTRODUCTION}

Abel [1] was the first to solve certain functional equations by reducing them to differential equations. Since then, differentiation has played an important role in the solution method (see, e.g., Aczel [2, 3]). Hilbert [4] in connection with the fifth of his famous problems decried the unnatural assumption of differentiability of the occurring functions. This led to investigations on the regularity of solutions of functional equations. Jarai $[5,6]$ showed that, for certain general functional equations, measurability implies continuity and continuity implies differentiability. Swiatak [7] and Tsutsumi and Haruki [8] showed that solutions of certain functional equations are $C^{\infty}$ by appealing to Hörmander's theorems on hypoellipticity [9]. Here, they relied on Schwartz's theory of distributions [10]. To my knowledge, Fenyö [11] was the first to use distributions to solve functional equations. Others who followed in this path are Neagu [12], Baker [13, 14], Koh [15], and Deeba and Koh [16-18]. In this paper, we discuss a method of reformulating certain functional equations in distributional forms. Since distributions are infinitely differentiable, this obviates the question of regularity. Our method consists of introducing distributional operators that mirror the functional operations such as addition, multiplication, scalar multiplication, and linear combination of the variables as well as the functions in the equation.

In this paper, we reformulate Hosszús functional equation

$$
f(x+y-x y)+g(x y)=h(x)+k(y)
$$

in the domain of distributions. (In fact, Hosszú's equation is simpler than equation (1), being the case where $f, g, h$, and $k$ are the same function.) We then show that the locally integrable solutions of (1) on an appropriate interval

Received by the editors July 13, 1992.

1991 Mathematics Subject Classification. Primary 46F10, 39B70. 
can be found using distribution theory. Our main theorem gives the correct general solution inadvertently missed by Fenyö [19].

\section{Notation}

Let $I$ be an open interval in $\mathbb{R}$, and let $I^{2}=I \times I \subset \mathbb{R}^{2}$. Let $\mathscr{D}(I)$ and $\mathscr{D}\left(I^{2}\right)$ be Schwartz spaces of $C^{\infty}$ functions with compact support on $I$ and $I^{2}$ respectively. Likewise, $\mathscr{E}(I)$ and $\mathscr{E}\left(I^{2}\right)$ are the spaces of $C^{\infty}$ functions on $I$ and $I^{2}$ respectively. We denote by $L_{\mathrm{loc}}(I)$ and $L_{\mathrm{loc}}\left(I^{2}\right)$ the spaces of equivalence classes of locally integrable functions on $I$ and $I^{2}$. The duals of these spaces are denoted by a prime, for example, $\mathscr{D}^{\prime}(I)$. Note that $\mathscr{D}(I) \subset$ $\mathscr{E}(I) \subset \mathscr{E}^{\prime}(I) \subset \mathscr{D}^{\prime}(I)$ (see [10]). The second inclusion will be interpreted by identifying the smooth function in $\mathscr{E}(I)$ with the regular distribution it generates in $\mathscr{E}^{\prime}(I)$. The topologies for these spaces will be the usual convergence concepts for $\mathscr{D}(I)$ and $\mathscr{E}(I)$ as given in [10] and the weak topologies for their duals. We denote the distribution corresponding to a locally integrable function $f$ by $\lambda_{f}$. If $f \in L_{\text {loc }}(I)$, then

$$
\left\langle\lambda_{f}, \phi\right\rangle=\int_{I} f(x) \phi(x) d x
$$

for any $\phi \in \mathscr{D}(I)$.

$D$ is the differentiation operator in $\mathscr{D}^{\prime}(I)$, whereas $D_{1}$ and $D_{2}$ are the partial differentiation operators in $\mathscr{D}^{\prime}\left(I^{2}\right)$ with respect to the first and second variable from $I^{2}$ respectively. These symbols will also denote the differentiation operators on subspaces of $\mathscr{D}^{\prime}$, for example, on $\mathscr{D}$.

\section{SOME LINEAR OPERATORS ON $\mathscr{D}^{\prime}$}

Let $E_{1}, E_{2}$ be integration operators from $\mathscr{D}\left(I^{2}\right)$ into $\mathscr{D}(I)$ given, respectively, by

$$
\begin{aligned}
& E_{1}[\phi](x)=\int_{I} \phi(x, y) d y, \\
& E_{2}[\phi](y)=\int_{I} \phi(x, y) d x
\end{aligned}
$$

for any $\phi \in \mathscr{D}\left(I^{2}\right)$. It is easy to see that these are continuous linear operators and we shall denote this fact by membership in $L\left[\mathscr{D}\left(I^{2}\right) ; \mathscr{D}(I)\right]$. Their adjoints $E_{i}^{*}(i=1,2)$ are operators from $\mathscr{D}^{\prime}(I)$ into $\mathscr{D}^{\prime}\left(I^{2}\right)$ defined by

$$
\left\langle E_{i}^{*}[T], \phi\right\rangle=\left\langle T, E_{i}[\phi]\right\rangle, \quad i=1,2,
$$

for $T \in \mathscr{D}^{\prime}(I)$ and $\phi \in \mathscr{D}\left(I^{2}\right)$. We note that $E_{i}^{*} \in L\left[\mathscr{D}^{\prime}(I) ; \mathscr{D}^{\prime}\left(I^{2}\right)\right]$.

Proposition 1 (see $[12,15])$. (a) If $f \in L_{\mathrm{loc}}(I), g(x, y)=f(x)$, and $h(x, y)$ $=f(y)$ for all $x, y \in I$, then $E_{1}^{*}[f]=g \in L_{\mathrm{loc}}\left(I^{2}\right)$ and $E_{2}^{*}[f]=h \in L_{\mathrm{loc}}\left(I^{2}\right)$.

(b) If $\alpha \in \mathscr{E}(I)$, then $E_{i}^{*}[\alpha] \in \mathscr{E}\left(I^{2}\right), i=1,2$.

(c) If $\alpha \in \mathscr{E}(I)$ and $T \in \mathscr{D}^{\prime}(I)$, then

$$
E_{i}^{*}[\alpha T]=E_{i}^{*}[\alpha] E_{i}^{*}[T], \quad i=1,2 .
$$

(d) If $T \in \mathscr{D}^{\prime}(I)$, then

$$
\begin{aligned}
& D_{1} E_{1}^{*}[T]=E_{1}^{*}[D T], \quad D_{1} E_{2}^{*}[T]=0, \\
& D_{2} E_{1}^{*}[T]=0, \quad D_{2} E_{2}^{*}[T]=E_{2}^{*}[D T] .
\end{aligned}
$$


Let $I$ be an interval that does not include the origin. Let $R$ be the operator from $\mathscr{D}\left(I^{2}\right)$ into $\mathscr{D}(I)$ given by

$$
R[\phi](x)=\int_{I} \frac{\phi(x / y, y)}{y} d y=\int_{I} \frac{\phi(y, x / y)}{y} d y
$$

for any $\phi \in \mathscr{D}\left(I^{2}\right)$. We note that $R \in L\left[\mathscr{D}\left(I^{2}\right) ; \mathscr{D}(I)\right]$. The adjoint of this operator is $R^{*} \in L\left[\mathscr{D}^{\prime}(I) ; \mathscr{D}^{\prime}\left(I^{2}\right)\right]$ defined by

$$
\left\langle R^{*}[T], \phi\right\rangle=\langle T, R[\phi]\rangle
$$

for any $\phi \in \mathscr{D}\left(I^{2}\right)$ and $T \in \mathscr{D}^{\prime}(I)$.

Proposition 2 (see [16], [17]). (a) If $f \in L_{\mathrm{loc}}(I)$, then $R^{*}[f] \in L_{\mathrm{loc}}\left(I^{2}\right)$ where $R^{*} f=f(x y)$.

(b) If $\alpha \in \mathscr{E}(I)$, then $R^{*}[\alpha] \in \mathscr{E}\left(I^{2}\right)$.

(c) If $\alpha \in \mathscr{E}(I)$ and $T \in \mathscr{D}^{\prime}(I)$, then

$$
\begin{gathered}
R^{*}[\alpha T]=R^{*}[\alpha] R^{*}[T], \\
D_{1}\left(R^{*}[T]\right)=E_{2}^{*}(\Omega) R^{*}(D T), \\
D_{2}\left(R^{*}[T]\right)=E_{1}^{*}(\Omega) R^{*}(D T),
\end{gathered}
$$

where $\Omega=t \in \mathscr{E}(I)$.

\section{Hosszú's FUNCTIONAL EQUATION IN DISTRIBUTIONS}

In 1969 Fenyö [19] solved Hosszú's equation (1) by means of distributions. He introduced a composition of three operators, two of which are of "faltung" type, that when applied to a regular distribution reduces it to $f(x+y-x y)$. We shall effect the same transformation by introducing a new operator $Z$ and taking its adjoint. Let $I=(0,1)$. Let $Z: \mathscr{D}\left(I^{2}\right) \rightarrow \mathscr{D}(I)$ be given by

$$
Z[\phi](x)=\int_{I} \frac{\phi((x-y) /(1-y), y)}{1-y} d y,
$$

and let its adjoint $Z^{*}: \mathscr{D}^{\prime}(I) \rightarrow \mathscr{D}^{\prime}\left(I^{2}\right)$ be given by

$$
\left\langle Z^{*}[T], \phi\right\rangle=\langle T, Z[\phi]\rangle=\langle T(x), Z[\phi](x)\rangle
$$

for any $\phi \in \mathscr{D}\left(I^{2}\right)$ and $T \in \mathscr{D}\left(I^{2}\right)$ and $T \in \mathscr{D}^{\prime}(I)$. It is easy to see that $Z \in L\left[\mathscr{D}\left(I^{2}\right) ; \mathscr{D}(I)\right]$ whereas $Z^{*} \in L\left[\mathscr{D}^{\prime}(I) ; \mathscr{D}^{\prime}\left(I^{2}\right)\right]$.

Proposition 3. (a) If $f \in L_{\mathrm{loc}}(I)$ and $g(x, y)=f(x+y-x y)$ for all $x, y \in I$, then $Z^{*}\left[\lambda_{f}\right]=\lambda_{g} \in L_{\mathrm{loc}}\left(I^{2}\right)$.

(b) If $\alpha \in \mathscr{E}(I)$, then $Z^{*}[\alpha] \in \mathscr{E}\left(I^{2}\right)$.

(c) If $\alpha \in \mathscr{E}(I)$ and $T \in \mathscr{D}^{\prime}(I)$, then

$$
Z^{*}[\alpha T]=Z^{*}[\alpha] Z^{*}[T] .
$$

(d) If $T \in \mathscr{D}^{\prime}(I)$, then

$$
\begin{aligned}
& D_{1} Z^{*}[T]=E_{2}^{*}[\theta] Z^{*}[D T], \\
& D_{2} Z^{*}[T]=E_{1}^{*}[\theta] Z^{*}[D T],
\end{aligned}
$$

where $\theta=1-t \in \mathscr{E}(I)$. 
Proof. (a)

$$
\begin{aligned}
\left\langle Z^{*}\left[\lambda_{f}\right], \phi\right\rangle & =\left\langle\lambda_{f}, \int_{I} \frac{\phi((x-y) /(1-y), y)}{1-y} d y\right\rangle \\
& =\int_{I^{2}} \frac{f(x) \phi((x-y) /(1-y), y)}{1-y} d y d x .
\end{aligned}
$$

Let $u=(x-y) /(1-y)$ and $v=y$. Noting that the Jacobian for this transformation is $1 /(1-y)$, we have

$$
d y d x /(1-y)=d u d v .
$$

Hence

$$
\begin{aligned}
\left\langle Z^{*}\left[\lambda_{f}\right], \phi\right\rangle & =\int_{I^{2}} f(u+v-u v) \phi(u, v) d u d v \\
& =\langle f(x+y-x y), \phi(x, y)\rangle .
\end{aligned}
$$

(b) This follows from (a) and the fact that partial differentiation under the integral sign is valid.

(c)

$$
\begin{aligned}
\left\langle Z^{*}[\alpha T], \phi\right\rangle & =\langle\alpha T, Z[\phi]\rangle=\left\langle T, \int_{I} \frac{\alpha(x) \phi((x-y) /(1-y), y)}{1-y} d y\right\rangle \\
& =\left\langle T, Z\left[Z^{*}[\alpha] \phi\right]\right\rangle=\left\langle Z^{*}[T], Z^{*}[\alpha] \phi\right\rangle=\left\langle Z^{*}[\alpha] Z^{*}[T], \phi\right\rangle .
\end{aligned}
$$

(d)

$$
\begin{aligned}
\left\langle D_{1} Z^{*}[T], \phi\right\rangle & =\left\langle Z^{*}[T],-D_{1} \phi\right\rangle \\
& =\left\langle T_{u},-\int_{1} \frac{1}{1-v} D_{1} \phi\left(\frac{u-v}{1-v}, v\right) d v\right\rangle \\
& =\left\langle T_{u},-\int_{I} \frac{\partial \phi}{\partial u}\left(\frac{u-v}{1-v}, v\right) d v\right\rangle \quad \text { (by the chain rule) } \\
& =\left\langle T_{u},-\frac{\partial}{\partial u} Z[(1-v) \phi]\right\rangle=\langle D T, Z[(1-v) \phi]\rangle \\
& =\left\langle Z^{*}[D T], E_{2}^{*}[\theta] \phi\right\rangle \quad(\text { by Proposition } 1(\mathrm{a})) \\
& =\left\langle E_{2}^{*}[\theta] Z^{*}[D T], \phi\right\rangle \quad(\text { where } \theta=1-t) .
\end{aligned}
$$

The second part is proved in an analogous way.

Definition 1. Let $F, G, H$, and $K$ belong to $\mathscr{D}^{\prime}(I)$. The equation

$$
Z^{*}[F]+R^{*}[G]=E_{1}^{*}[H]+E_{2}^{*}[K]
$$

is called the Hosszú equation in distributions.

Proposition 4. If $F, G, H$, and $K$ are regular distributions, i.e., locally integrable functions $f, g, h$ and $k$, then equation (19) reduces to the Hosszu functional equation (1).

Proof. The proof follows from Propositions 1, 2, and 3. 
We now solve equation (19). We first note that from Proposition 2, for $T \in \mathscr{D}^{\prime}(I)$,

$$
\begin{aligned}
D_{1} D_{2} & R^{*}[T]=D_{1} E_{1}^{*}[\Omega] R^{*}[D T]=D_{1} x R^{*}[D T] \\
& =R^{*}[D T]+x E_{2}^{*}[\Omega] R^{*}\left[D^{2} T\right]=R^{*}[D T]+x y R^{*}\left[D^{2} T\right] \\
& \left.=R^{*}[D T]+R^{*}[t] R^{*}\left[D^{2} T\right] \quad \text { (by virtue of Proposition } 2(\mathrm{a})\right) \\
& =R^{*}\left[D T+t D^{2} T\right]=R^{*}[D t D T] .
\end{aligned}
$$

Similarly, from Proposition 3, for $T \in \mathscr{D}^{\prime}(I)$,

$$
\begin{aligned}
D_{1} D_{2} & Z^{*}[T]=D_{1} E_{1}^{*}[\theta] Z^{*}[D T]=D_{1}(1-x) Z^{*}[D T] \\
& =-Z^{*}[D T]+(1-x) E_{2}^{*}[\theta] Z^{*}\left[D^{2} T\right] \\
& =-Z^{*}[D T]+(1-x)(1-y) Z^{*}\left[D^{2} T\right] \\
& =-Z^{*}[D T]+[1-x-y+x y] Z^{*}\left[D^{2} T\right] \\
& =-Z^{*}[D T]+Z^{*}[1-t] Z^{*}\left[D^{2} T\right] \quad(\text { by virtue of Proposition } 3(\mathrm{a})) \\
& =Z^{*}\left[-D T+(1-t) D^{2} T\right]=Z^{*}[D(1-t) D T] .
\end{aligned}
$$

Applying $D_{1} D_{2}$ on (19) and using the properties (6), (7), (20), and (21), we have

$$
Z^{*}[D(1-t) D F]+R^{*}[D t D G]=0 .
$$

Applying $D_{1}$ and $D_{2}$ on (22), we obtain by virtue of Propositions 2 and 3 ,

$$
\begin{aligned}
& (1-y) Z^{*}\left[D^{2}(1-t) D F\right]+y R^{*}\left[D^{2} t D G\right]=0, \\
& (1-x) Z^{*}\left[D^{2}(1-t) D F\right]+x R^{*}\left[D^{2} t D G\right]=0 .
\end{aligned}
$$

Multiplying $(23)$ by $(1-x)$ and $(24)$ by $(1-y)$ and subtracting one from the other yields

$$
(y-x) R^{*}\left[D^{2} t D G\right]=0 .
$$

For $\phi(x, y) \in \mathscr{D}\left(I^{2}\right), \operatorname{supp}[(y-x) \phi(x, y)] \subset I^{2}$. Thus $R[(y-x) \phi(x, y)] \in$ $\mathscr{D}(I)$ and equation (25) implies that

$$
D^{2} t D G=0 .
$$

Indeed, since $R$ is a surjective map onto $D(I)$,

$$
\begin{aligned}
\left\langle(y-x) R^{*}\left[D^{2} t D G\right], \phi\right\rangle & =\left\langle D^{2} t D G, R(y-x) \phi\right\rangle \\
& =\left\langle D^{2} t D G, \psi(x)\right\rangle=0 \text { for every } \psi \in \mathscr{D}(I) .
\end{aligned}
$$

The differential equation (26) is solved by

$$
G=c_{1} t+c_{2} \ln t+c_{3} \text {. }
$$

From (22), (26), and the fact that $Z$ is also a surjection, we have

$$
D^{2}(1-t) D F=0 \text {. }
$$

This differential equation is solved by

$$
F=\alpha_{1} t+\alpha_{2} \ln (1-t)+\alpha_{3} .
$$

Since the distributional solutions given by (27) and (29) are locally integrable functions on $I$, we may substitute them into the left-hand side of (1), giving

$$
\alpha_{1}(x+y-x y)+\alpha_{2} \ln (1-x-y+x y)+\alpha_{3}+c_{1} x y+c_{2} \ln x y+c_{3} \text {. }
$$

Thus, (3) can be written in the form $h(x)+k(y)$ if and only if $\alpha_{1}=c_{1}$. We have proved the following theorem. 
Theorem 1. If $F, G, H, K \in \mathscr{D}^{\prime}(I)$ satisfy equation (19), then they are regular distributions generated by the functions $f(t)=c t+\alpha \ln (1-t)+m, g(t)=$ $c t+\beta \ln t+l, h(t)=c t+\alpha \ln (1-t)+\beta \ln t+r$, and $k(t)=c t+\alpha \ln (1-t)+\beta \ln t+s$ where $c, \alpha$, and $\beta$ are arbitrary constants and $m+l=r+s$ hold.

It is noteworthy that Fenyö's result [19, Theorem 2] is not quite correct. He had erroneously arrived at $\alpha=\beta=0$.

The case of Hosszú's original equation

$$
f(x+y-x y)+f(x y)=f(x)+f(y)
$$

can be written in our distributional setting as

$$
Z^{*}[F]+R^{*}[F]=E_{1}^{*}[F]+E_{2}^{*}[F] .
$$

We now have the following corollaries.

Corollary 1. If $F$ is a regular distribution, i.e., a locally integrable function $f$, then equation (32) reduces to equation (31).

Corollary 2. If $F \in \mathscr{D}^{\prime}(I)$ satisfies (32), then $F$ is a regular distribution generated by the function $f(t)=c t+m$, where $c$ and $m$ are arbitrary constants.

Proof. As in the arguments leading to Theorem 1, we have

$$
F=c_{1} t+c_{2} \ln t+c_{3} \text {. }
$$

Thus, the distributional solution of (32) is given by (33) which is a locally integrable function on $I$. Since (32) reduces to (31) for regular distributions, we may substitute (33) into (31) to see that $c_{2}=0$.

Concluding remarks. Another approach to the distributional analogue for the Hosszú equation is via the use of pullbacks. A theorem of Hörmander [9, p. 134] guarantees the existence of a unique continuous linear map $T^{*}$ for a diffeomorphism $T$ such that $T^{*} u=u \circ T$ when $u \in \mathscr{D}(I)$, for example. In the present context, $T: f(x) \rightarrow f(x+y-x y)$ is such a diffeomorphism from $\mathscr{D}(I)$ onto $\mathscr{D}\left(I^{2}\right)$. Thus $T^{*}$ would correspond to our operator $Z^{*}$. Similarly, our operator $R^{*}$ will have its corresponding pullback. These ideas are exploited by Baker in a paper on the Aczél-Chung equation (see [20]). It would be interesting to study the connection between pullback maps and the adjoint operators we use.

\section{REFERENCES}

1. N. H. Abel, Über die Funktionen die der Gleichung $\phi x+\phi y=\phi(x f y+y f x)$ genug thun, J. Reine Angew. Math. 2 (1827), 386-394.

2. J. Aczel, Grundrißeiner allgemeinen Behandlung von einigen Funktionalgleichungstypen, Publ. Math. Debrecen 3 (1953), 119-132.

3. $\ldots$, Lectures on functional equations and their applications, Academic Press, New York, 1966.

4. D. Hilbert, Mathematische probleme, Nachr. Ges. Wiss. Göttingen (1900), 253-297; Proc. Sympos. Pure Math., vol. 38, Amer. Math. Soc., Providence, RI, 1976, p. 14.

5. A. Jarai, On regular solutions of functional equations, Aequationes Math. 30 (1986), 21-54.

6. __ Regularity property of functional equations, Aequationes Math. 25 (1982), 52-66.

7. H. Swiatak, On the regularity of the distributional and continuous solutions of the functional equation $\sum_{i=1}^{k} a_{i}(x, t) f\left(x+\phi_{i}(t)\right)=b(x, t)$, Aequationes Math. 1 (1968), 6-19. 
8. A. Tsutsumi and S. Haruki, The regularity of solutions of functional equations and hypoellipticity, Functional Equations, History, Applications \& Theory, Reidel, Boston, MA, 1984, pp. 99-112.

9. L. Hörmander, The analysis of linear partial differential operators. I, Springer Verlag, Berlin and New York, 1983.

10. L. Schwartz, Théorie des distributions, Hermann, Paris, 1966.

11. I. Fenyö, Über eine lösungsmethode gewisser funktionalgleichungen, Acta Math. Hungar 7 (1957), 383-396.

12. M. Neagu, About the Pompeiu equation in distributions, Inst. Politehn. "Traian Vuia" Timisoara. Lucrăr. Sem. Mat. Fiz. 1984, 62-66.

13. J. A. Baker, Functional equations, tempered distributions and Fourier transforms, Trans. Amer. Math. Soc. 315 (1989), 57-68.

14. $\ldots$ Functional equations, distributions and approximate identities, Canad. J. Math. 42 (1990), 696-708.

15. E. L. Koh, The Cauchy functional equations in distributions, Proc. Amer. Math. Soc. 106 (1989), 641-647.

16. E. Y. Deeba and E. L. Koh, The Pexider functional equations in distributions, Canad. J. Math. 42 (1990), 304-314.

17. , D'Alembert functional equations in distributions, Proc. Amer. Math. Soc. 116 (1992), 157-164.

18. _ Coupled functional equations in distributions, Indian J. Math., Singh Memorial Volume 33 (1991), 275-285.

19. I. Fenyö, On the general solution of a functional equation in the domain of distributions, Aequationes Math. 3 (1969), 236-246.

20. J. A. Baker, On a functional equation of Aczél and Chung, Aequationes Math. (to appear).

Department of Mathematics and Statistics, University of Regina, Regina, Sashatchewan, Canada S45 0A2

E-mail address: elkoh@max.cc.uregina.ca 